\title{
ÁLGEBRA ISLÂMICA DE AL-KHWARIZMI A OMAR KHAYYAM
}

\author{
ISLAMIC ALGEBRA FROM AL-KHWARIZMI TO OMAR KHAYYAM
}

\author{
Rosângela Araújo da Silva ${ }^{1}$; Bernadete Barbosa Morey ${ }^{2}$
}

\begin{abstract}
RESUMO
A álgebra das equações tem seus primórdios na ciência islâmica medieval. Este trabalho é um recorte de uma pesquisa doutoral mais ampla em desenvolvimento que envolve a análise do tratado algébrico de Omar Khayyam (1048-1131), cujo título é Al-Risala fi-l-barahin 'ala masa'il al-jabr wa-l-muqabala (Tratado sobre demonstrações dos problemas da álgebra e almuqabala). Ao se tratar de álgebra das equações, torna-se imprescindível citar o sábio al-Khwarizmi (780850), que escreveu um tratado algébrico, conhecido até os dias de hoje como a Álgebra de alKhwarizmi (780-850). O presente trabalho é sobre o desenvolvimento da álgebra islâmica de al-Khwarizmi a Omar Khayyam. O estudo tem como objetivo descrever o que foi elaborado e desenvolvido em álgebra islâmica de al- Khwarizmi a Omar Khayyam. Possibilitando um melhor entendimento do significado da álgebra de Khayyam, pois em seu tratado ele menciona alguns sábios que estudaram álgebra antes dele. A pesquisa foi realizada por meio de pesquisa bibliográfica, a princípio na plataforma de periódicos da Capes e para complementar utilizamos a busca pelas biografias dos sábios para descrever a trajetória, na página eletrônica Mac Tutor. Apresentamos este percurso de desenvolvimento da álgebra, como resultado obtido, considerando que novos estudos podem ampliá-lo. Os estudiosos que encontramos foram: AlKhwarizmi resolvendo equações lineares e quadráticas, apresentando a álgebra como uma prática para resolver problemas e nomeando a álgebra; al-Mahani que chegou em uma equação envolvendo cubos, quadrados e números; Abu Kamil que aplicou a álgebra no pentágono e no decágono regular; al-Karaji que aritmetizou a álgebra, primórdios para o modelo da álgebra moderna; e chegando a Omar Khayyam que desenvolveu por meio de intersecções de seções cônicas um método geométrico de resolver equações cúbicas.
\end{abstract}

Palavras-chave: Álgebra; Al-Khwarizmi; Desenvolvimento; Equações; Omar Khayyam.

\begin{abstract}
Equation algebra has its beginnings in medieval Islamic science. This work is an excerpt from a broader doctoral research under development that involves the analysis of the algebraic treatise
\end{abstract}

\footnotetext{
${ }^{1}$ Mestre em Matemática pela Universidade Federal do Ceará (UFC). Doutoranda em Ensino de Ciências e Matemática pela Universidade Federal do Rio Grande do Norte (UFRN). Professora no Instituto Federal do Rio Grande do Norte (IFRN), Campus Santa Cruz, Rio Grande do Norte, Brasil. Endereço para correspondência: Rua São Braz, 304, Bairro Paraíso, Santa Cruz, RN, Brasil, CEP: 59200-000. E-mail: rosangelasilva@ifrn.edu.br.

(iD) ORCID iD: https://orcid.org/0000-0002-9174-6232.

2 Doutora em Educação Matemática pela Universidade Amizade dos Povos. Pós-doutora na UNESP-RC em História da Matemática, Pós-doutora na Universidade de Penza, Rússia em História da Matemática. Pós-doutora na Laurentian University, Sudbury, Canadá. Endereço para correspondência: Rua Coronel João Medeiros, 1850 - Lagoa Nova, Natal, RN, Brasil, CEP: 59077-000. E-mail: bernadetemorey@gmail.com.
}

(iD) ORCID iD: https://orcid.org/0000-0003-3253-0383. 
by Omar Khayyam (1048-1131), whose title is Al-Risala fi-l-barahin 'ala masa'il al-jabr wa- 1muqabala (Treaty on demonstrations of the problems of algebra and almuqabala). When dealing with the algebra of the equations, it is essential to quote the sage al-Khwarizmi (780-850), who wrote an algebraic treatise, known until today as the Algebra of al-Khwarizmi (780-850). The present work is about the development of Islamic algebra from al-Khwarizmi to Omar Khayyam. The study aims to describe what was developed and developed in Islamic algebra from alKhwarizmi to Omar Khayyam. Enabling a better understanding of the meaning of Khayyam algebra, as in his treatise he mentions some scholars who studied algebra before him. The research was carried out by means of bibliographic research, at first on the platform of Capes journals and to complement, we used the search for the biographies of the sages to describe the trajectory, on the Mac Tutor website. We present this algebra development path, as a result obtained, considering that new studies can expand it. The scholars we encountered were: Al-Khwarizmi solving linear and quadratic equations, presenting algebra as a practice for solving problems and naming algebra; al-Mahani who arrived at an equation involving cubes, squares and numbers; Abu Kamil who applied algebra to the pentagon and regular decagon; al-Karaji who arithmeticized algebra, beginnings for the modern algebra model; and reaching Omar Khayyam who developed, through intersections of conic sections, a geometric method of solving cubic equations.

Keywords: Algebra; Al-Khwarizmi; Development; Equations; Omar Khayyam. 


\section{Introdução}

O presente trabalho trata do desenvolvimento da álgebra islâmica de alKhwarizmi a Omar Khayyam. Esta pesquisa foi motivada mediante as necessidades de uma pesquisa doutoral em desenvolvimento, que incluem a análise do tratado algébrico de Omar Khayyam (1048-1131), cuja temática é a álgebra das equações.

Ao tratarmos de álgebra das equações, torna-se imprescindível citar o sábio alKhwarizmi (780-850), reconhecido como um dos primeiros estudiosos da álgebra. Assim, surge a necessidade de escrevermos sobre quais obras contribuíram para o desenvolvimento da álgebra no interstício entre os estudiosos al-Khwarizmi e Omar Khayyam.

A investigação foi realizada por meio de uma pesquisa bibliográfica constituindose especialmente de livros e artigos científicos (GIL, 2008).

O presente texto apresenta, afora essa breve introdução, uma seção acerca das publicações sobre o desenvolvimento da álgebra islâmica medieval e outra seção na qual apresentamos os autores e as obras em álgebra no intervalo de tempo em questão, finalizando com as considerações alcançadas.

\section{Pesquisas sobre o desenvolvimento da álgebra islâmica medieval}

Uma das contribuições da ciência islâmica medieval foi a criação e o desenvolvimento da álgebra das equações. O Núcleo de Pesquisa em História e Educação Matemática (NuPHEM) ${ }^{3}$ que tem produzido uma série de estudos sobre a matemática islâmica, tem-se voltado agora para o estudo da álgebra.

Um dos recortes de uma pesquisa mais ampla inclui a análise do tratado algébrico de Omar Khayyam (1048-1131), intitulado Al-Risala fi-l-barahin 'ala masa'il al-jabr wal-muqabala (Tratado sobre as demonstrações dos problemas da álgebra e almuqabala). É fato bastante conhecido que um dos primeiros estudiosos islâmicos de renome foi alKhwarizmi que escreveu no início do século IX. uma obra que ficou conhecida como a Álgebra de al-Khwarizmi (780-850). Havia então um intervalo em torno de 200 anos que precisava ser investigado, nem que fosse em linhas gerais, com relação aos trabalhos realizados em álgebra pelos estudiosos islâmicos. Esclarecer os acontecimentos em

\footnotetext{
${ }^{3}$ Núcleo de pesquisa do Programa de Pós-Graduação em Ensino de Ciências e Matemática da Universidade Federal do Rio Grande do Norte (UFRN).
} 
álgebra nesse intervalo era importante para entender melhor o significado e o valor da álgebra de Omar Khayyam.

Inicialmente realizamos uma busca nos periódicos na plataforma da Capes ${ }^{4}$. A busca foi realizada usando as possíveis combinações, em português e em inglês, dos descritores: "desenvolvimento da álgebra islâmica", "desenvolvimento da álgebra árabe”, “desenvolvimento da álgebra medieval" e outras variações. Os resultados da busca foram poucos e insatisfatórios, pois, os artigos indicados não respondiam à nossa questão da álgebra de al-Khwarizmi a Khayyam.

O artigo publicado em 2005, na revista Historia Mathematica, intitulado Mal, enunciations, and the prehistory of Arabic álgebra dos autores Jeffrey A. Oaks e Haitham M. Alkhateeb, essa pesquisa trata das diferentes formas como a palavra mal $^{5}$ é utilizada nos livros de al-Khwarizmi, Abu Kamil e Ibn Badr. Dessa forma não respondeu diretamente à nossa questão, mas, ao citar alguns nomes, já deu algumas pistas.

Ainda permanecendo a necessidade de escrever sobre a álgebra islâmica até Khayyam optamos por outro caminho: examinar as biografias de estudiosos islâmicos do período em questão e destacar os que produziram trabalhos em álgebra. Uma boa fonte de biografias é a plataforma digital Mac Tutor ${ }^{6}$ da Escola de Matemática e Estatística da Universidade de St. Andrews, na Escócia. Inicialmente, usamos os nomes citados por Omar Khayyam no tratado algébrico e as indicações do texto de Oaks e Alkhateeb (2005), e aos poucos fomos encontrando as biografias de outros sábios.

$\mathrm{Na}$ próxima sessão apresentaremos o percurso da álgebra islâmica de alKhwarizmi a Omar Khayyam.

\section{O caminho da álgebra islâmica de al-Khwarizmi a Omar Khayyam}

Os estudos nos mostram os seguintes estudiosos que contribuíram para o percurso da álgebra islâmica, no intervalo destacado em nossa pesquisa, conforme figura 1 .

\footnotetext{
${ }^{4}$ A busca foi realizada de 15 a 20 de fevereiro de 2021.

5 Mal é o termo árabe usado para designar o quadrado do 'desconhecido' ou da 'coisa', atualmente denominamos o 'desconhecido' ou a 'coisa' por incógnita ou $\mathbf{x}$.

${ }^{6}$ Página eletrônica: https://mathshistory.st-andrews.ac.uk/Biographies/
} 
Figura 1 - Caminho da álgebra islâmica de al-Khwarizmi a Omar Khayyam

\section{Al-Khwarizmi (780-850)}

- O livro sobre o cálculo de álgebra e almuqabala

\section{Al-Mahani (820-880)}

- Equação envolvendo cubos, quadrados e números

\section{Abu Kamil Shuja (850-??)}

- O Livro sobre álgebra e almuqabala

\section{Al-Karaji (953-1029)}

- Tratado algébrico Al-Fakhri

\section{Omar Khayyam (1048-1131)}

- Tratado sobre as demonstrações dos problemas da álgebra e almuqabala

Fonte: Elaborado pelas autoras (2021).

Em sequência, descreveremos um pouco sobre cada um deles e suas contribuições para o desenvolvimento da álgebra.

- Abu Ja'far Muhammad ibn Musa al-Khwarizmi (em torno de 780-850) sábio islâmico, cujo tratado algébrico do início do século IX intitulado Al-Kitab al-muhtasar fi hisab al-jabr wa-l-muqabala (O livro sobre o cálculo de álgebra e almuqabala), conhecido simplesmente como a Álgebra de al-Khwarizmi. Em sua álgebra al-Khwarizmi apresenta as duas operações de al-jabr e al-muqabala, resolvendo seis tipos de equações lineares e quadráticas. Youschkevitch (1976), Rashed (1994) e Berggren (2003), islamistas reconhecidos, o consideram o precursor da Álgebra (em uma historiografia em que a noção de precursor faça sentido), no momento em que a nomeou e tornou-a uma disciplina autônoma.

Al-Khwarizmi apresenta a álgebra de forma prática, resolvendo problemas de heranças (O'CONNOR; ROBERTSON, 1999c). Entre as álgebras do período islâmico medieval, a de al-Khwarizmi é a mais conhecida, tendo sido traduzida em vários idiomas, traremos duas delas, cujas referências bibliográficas são: 
O texto da tradução para a língua inglesa em 1831 trata o sábio al-Khwarismi como Mohammed ben Musa. A obra foi nomeada por The álgebra of Mohammed bem Musa, editada e traduzida pelo alemão Friedrich August Rosen (1805-1837) mais conhecido como Frederic Rosen, impressa pelo Fundo de Tradução Oriental, em Londres (ROSEN, 1831).

$\checkmark \mathrm{Na}$ tradução para a língua espanhola a obra foi intitulada de El libro del Álgebra, realizada por Ricardo Moreno Castillo, na cidade de Madrid, pela editora Nivola libros y ediciones, em 2009. Observamos que a grafia do nome do sábio em espanhol é al-Jwarizmi (JWARIZMI, 2009).

Na figura 2, trazemos as ilustrações das traduções para o inglês no século XIX e para o espanhol no século XXI.

Figura 2 - Traduções da Álgebra de al-Khwarizmi

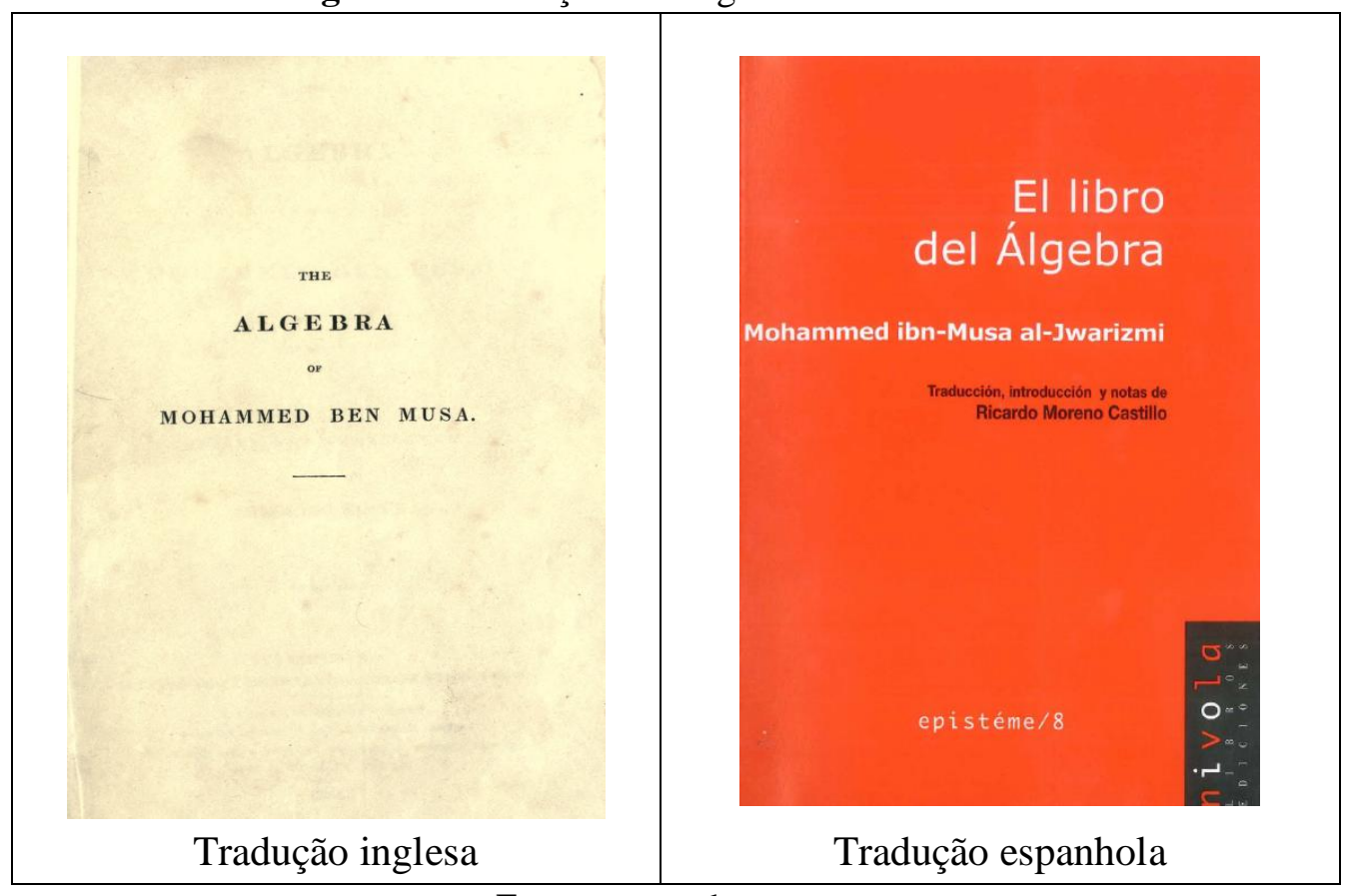

Fonte: acervo das autoras

- Abu Abd Allah Muhammad ibn Isa al-Mahani (em torno de 820-880) foi um estudioso islâmico que tentou resolver alguns dos problemas de Arquimedes sobre o corte de esferas. O problema de Arquimedes que foi tentado por ele, de uma nova forma, foi cortar uma esfera por um plano de modo que as duas partes resultantes tivessem volumes em uma determinada proporção. 
No tratado algébrico de Omar Khayyam há citações ao trabalho desenvolvido em álgebra por al-Mahani. Al-Mahani chegou a uma equação envolvendo cubos, quadrados e números, porém não conseguiu resolver (O'CONNOR; ROBERTSON, 1999a).

- Abu Kamil Shuja ibn Aslam ibn Muhammad ibn Shuja (em torno de 850-??) era um matemático que O'Connor e Robertson (1999d) o consideram um dos sucessores de al-Khwarismi. Abu Kamil era conhecido como al-Hasib al-Misri, que significa a calculadora do Egito, o que reforça sua procedência egípcia.

Abu Kamil aplicou métodos algébricos a problemas geométrico no livro Kitàb alJabr wa-al-muqâbala (O Livro sobre álgebra e almuqabala) dividido em três partes, sejam elas: Soluções de equações do segundo grau; aplicações da álgebra para o pentágono e o decágono regular; e equações diofantinas e problemas de matemática recreativa.

- Abu Bekr ibn Muhammad ibn al-Husayn al-Karaji (953- em torno de 1029) foi um matemático islâmico que viveu em Bagdá a maior parte de sua vida e seu tratado algébrico denominado Al-Fakhri, recebeu essa nomeação em homenagem ao governante de Bagdá chamado Fakhr.

O texto de al-Karaji é considerado uma aritmetização da álgebra. O'Connor e Robertson (1999b) afirmam que ele pode ser considerado o primeiro a libertar a álgebra das operações geométricas e conseguiu substituí-las pelo modelo de operações que evoluíram para o modelo usado na álgebra atualmente.

- Mais conhecido no ocidente como Omar Khayyam (1048-1131), o poeta, astrônomo e matemático Ghiyath al-Din Abu'l-Fath Umar ibn Ibrahim Al-Nisaburi alKhayyam nasceu em 15 de maio de 1048, em Nishapur na Pérsia (atualmente o Irã) e faleceu na mesma cidade em 4 de dezembro de 1131 (YOUSCHKEVITCH; ROSENFELD, 2008).

Sua trajetória de vida o levou a outras cidades, entre elas, a cidade de Samarcanda no Uzbequistão em 1070 (figura 3), onde foi apoiado pelo jurista Abu Tahir. Momento que lhe permitiu escrever sua obra de álgebra mais famosa, Al-Risala fi-l-barahin 'ala masa'il al-jabr wa-l-muqabala (Tratado sobre as demonstrações dos problemas da álgebra e almuqabala). 
Figura 3 - Mapa da região de Nishapur e Samarcanda

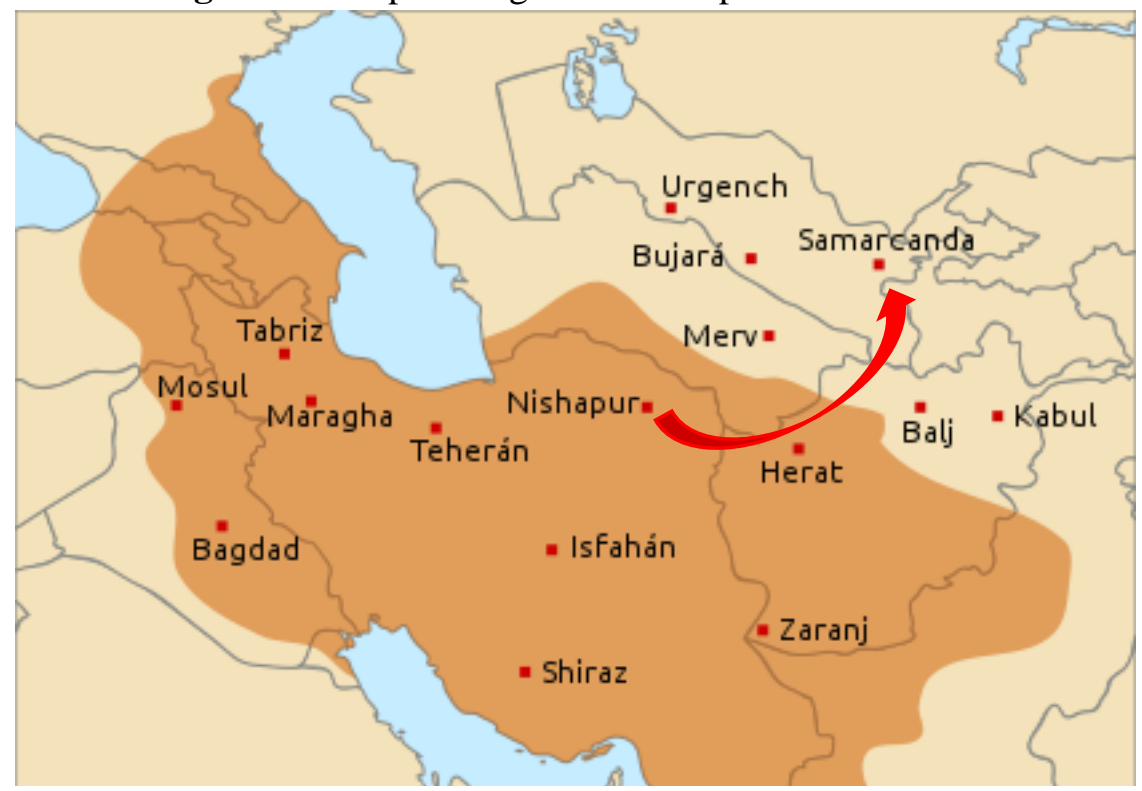

Fonte: https://commons.wikimedia.org/wiki/File:Afsharid_(1736-1802)-es.svg

O Tratado Algébrico de Omar Khayyam traz 25 tipos de equações e, em sequência, ele apresenta as resoluções de cada um desses tipos. Podemos dividir em 11 tipos resolvidos por métodos euclidianos e 14 tipos de equações cúbicas com solução por interseções de seções cônicas. Na figura 4 apresentamos uma página do tratado com uma ilustração da solução geométrica de uma equação cúbica.

Figura 4 - Página do manuscrito original

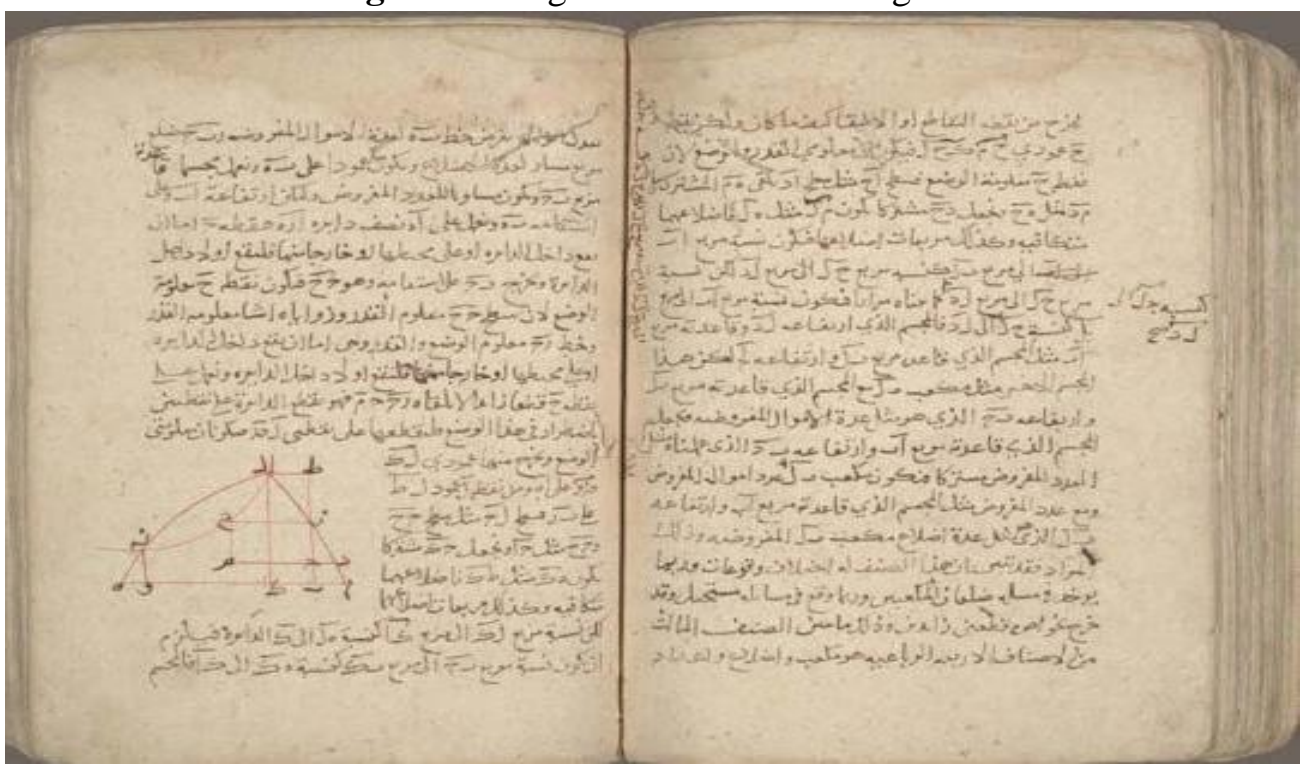

Fonte: https://www.maa.org/press/periodicals/convergence/mathematical-treasures-omar-khayyamsalgebra 
Omar Khayyam desenvolveu em seu tratado algébrico um método geométrico de resolver equações cúbicas, por meio de intersecções de seções cônicas e, dessa forma, até o momento histórico, foi o primeiro a conceber uma teoria geral das equações cúbicas. Outro ponto interessante nesse tratado foi o uso da linguagem retórica, ele não utilizou símbolos, nem mesmo para os números (ROSENFELD, 1953).

Posteriormente Malik-Shah, governante de Esfahan, neto de Toghril Beg, fundador da dinastia Seljuq, convidou Khayyam para construir um Observatório em Esfahan e liderar o corpo de estudiosos deste observatório.

Khayyam permaneceu em Esfahan por 18 anos e ali produziu tantos outros trabalhos, entre esses estudos Khayyam liderou as atividades para a compilação de tabelas astronômicas e também contribuiu para a reforma do calendário em 1079, cuja medição do ano foi apresentada com a duração de 365,24219858156 dias (O'CONNOR; ROBERTSON, 1999e).

Como poeta Khayyam escreveu o Rubaiyat, e em Matemática além do tratado mais ilustre, ele também escreveu: Problemas de Aritmética e Comentários sobre os postulados difíceis do livro de Euclides.

\section{Considerações Finais}

O presente trabalho surgiu da necessidade de escrever sobre a álgebra islâmica de al-Khwarizmi a Omar Khayyam. Neste intuito recorremos a uma pesquisa de publicações, que nos respondeu parcos e insatisfatórios resultados.

Assim, usando as indicações dadas no próprio texto de Omar Khayyam e pelo artigo de Oaks e Alkhateeb (2005) fomos pesquisar as biografias dos estudiosos citados, e como resultado conseguimos o intento inicial do estudo.

Apresentamos o percurso do desenvolvimento da álgebra islâmica desde alKhwarizmi até Omar Khayyam, os seguintes sábios contribuíram:

$\checkmark$ Al-Khwarizmi nomeando a álgebra com o seu tratado Al-Kitab al-muhtasar $f i$ hisab al-jabr wa-l-muqabala (O livro sobre o cálculo de álgebra e almuqabala), resolvendo equações lineares e quadráticas, e apresentando a álgebra como uma prática para resolver problemas. 
$\checkmark$ Al-Mahani que tentou resolver alguns problemas de Arquimedes e chegou em uma equação envolvendo cubos, quadrados e números, mas não conseguiu resolver.

$\checkmark$ Abu Kamil que usou métodos algébricos a problemas geométricos, com aplicações da álgebra para o pentágono e o decágono regular.

$\checkmark$ Al-Karaji realizou em seu texto uma aritmetização da álgebra, o primeiro passo para o modelo usado na álgebra moderna.

$\checkmark$ Omar Khayyam no seu tratado algébrico desenvolveu um método geométrico de resolver equações cúbicas, por meio de intersecções de seções cônicas, e apresentou soluções para todas as equações cúbicas.

Ressaltamos como é importante observarmos o desenvolvimento das ideias e a elaboração de novos conhecimentos. Apresentamos o percurso do desenvolvimento da álgebra de al-Khwarizmi a Omar Khayyam, e consideramos que novos estudos podem modificá-lo, pois uma pesquisa é construída todos os dias.

\section{Referências}

BERGGREN, J. L. Episodes in the mathematics of medieval islam. New York: Springer-Verlag Inc., 2003.

GIL, A. C. Métodos e técnicas de pesquisa social. 6. ed. São Paulo: Atlas, 2008.

JWARIZMI, M. M. El libro del Álgebra. Tradução: Ricardo Moreno Castillo. Madrid: Nivola libros y ediciones, 2009.

OAKS, J. A.; ALKHATEEB, H. M. Mal, enunciations, and the prehistory of Arabic álgebra. Historia Mathematica, n. 32, p. 400-425, 2005. Disponível em: https://www.mendeley.com/catalogue/207db5a5-0d07-393b-95b5-e64c81389c3d/. Acesso em: 20 fev. 2021.

O'CONNOR, J. J.; ROBERTSON, E. F. Abu Abd Allah Muhammad ibn Isa AlMahani. St. Andrews: University St. Andrews, 1999a. Disponível em: https://mathshistory.st-andrews.ac.uk/Biographies/Al-Mahani/. Acesso em: $24 \mathrm{fev}$. 2021.

O'CONNOR, J. J.; ROBERTSON, E. F. Abu Bekr ibn Muhammad ibn al-Husayn Al-Karaji. St. Andrews: University St. Andrews, 1999b. Disponível em: https://mathshistory.st-andrews.ac.uk/Biographies/Al-Karajil. Acesso em: 24 fev. 2021.

O'CONNOR, J. J.; ROBERTSON, E. F. Abu Ja'far Muhammad ibn Musa AlKhwarizmi. St. Andrews: University St. Andrews, 1999c. Disponível em: 
https://mathshistory.st-andrews.ac.uk/Biographies/Al-Khwarizmi/. Acesso em: 24 fev. 2021.

O'CONNOR, J. J.; ROBERTSON, E. F. Abu Kamil Shuja ibn Aslam ibn

Muhammad ibn Shuja. St. Andrews: University St. Andrews, 1999d. Disponível em: https://mathshistory.st-andrews.ac.uk/Biographies/Abu_Kamil/. Acesso em: 24 fev. 2021.

O'CONNOR, J. J.; ROBERTSON, E. F. Omar Khayyam. St. Andrews: University St. Andrews, 1999e. Disponível em: https://mathshistory.standrews.ac.uk/Biographies/Khayyam/. Acesso em: 24 fev. 2021.

RASHED, R. The development of Arabic mathematics: between arithmetic and algebra. Tradução: Angela Armstrong. Boston: Springer, 1994.

ROSEN, F. The algebra of Mohamed ben Musa. Londres: Impresso pelo Fundo de tradução oriental, 1831.

ROSENFELD, B. Математические Tpaktatы Омара Хайяма. Историкоматематика Исследования, Moscou, n. 06, p. 11-66, 1953.

YOUSCHKEVITCH, A. P. Les Mathématiques Arabes: VIIIe-XVe siècles. Trad.: M. Cazenazeet K. Jaouiche. Paris: VRIN, 1976.

YOUSCHKEVITCH, A. P.; ROSENFELD, B. A. Al-Khayyām - Complete Dictionary of Scientific Biography. 2008. Disponível em: https://mathshistory.standrews.ac.uk/DSB/Khayyam.pdf. Acesso em: 24 fev. 2021.

Recebido em: 27 / 02 / 2021

Aprovado em: 16 / 04 / 2021 